Conclusions: There was established that positive response on therapy with denosumab in BMD in NH and DF is associated with RF-positivity. The particular contribution to the negative response in L1-L4 and $\mathrm{HN}$ is associated with GC intake (previous intake more than 3 months in the anamnesis), purpose of the GC after menopause onset. There was note that the increase of erosion score is associated with either lower BMD in L1-L4 (at baseline and after treatment) or back correlation with $\mathrm{BMD}$ increase in DF. Also the higher cumulative GC dose and back correlation with BAP base level were observed at patients with increased erosion score. The only factors that we could reveal in patients with increased JSN score were surgical menopause in anamnesis and lower value of BMD dynamics (\%) in DF after treatment. In general it was a direct correlation between erosion score and JSN score.

Disclosure of Interest: None declared

DOI: 10.1136/annrheumdis-2018-eular.5153

\section{AB0355 THE RELATIONSHIP BETWEEN HAND PREHENSILE STRENGTH, CLINICAL ACTIVITY AND FUNCTIONAL CAPACITY IN PATIENTS WITH RHEUMATOID ARTHRITIS}

I.P. Guzmán Guzmán ${ }^{1}$, J.E. Navarro-Zarza², G.A. Alonso-Silverio ${ }^{3}$, A. AlarcónParedes $^{3}$, D. Jerez-Mayorga ${ }^{4}$, I. Parra-Rojas ${ }^{1} .{ }^{1}$ Facultad de Ciencias Químico Biológicas, Universidad Autónoma de Guerrero; ${ }^{2}$ Hospital General; ${ }^{3}$ Facultad de Ingeniería, Universidad Autónoma de Guerrero, Chilpancingo, Mexico; ${ }^{4} \mathrm{Facultad}$ de Ciencias de la Rehabilitación, Universidad Andres Bello, Santiago, Chile

Background: The hand is an anatomical structure with a large number of joints; its prehensile grasp capability constitutes a highly specialised biomechanical function. In rheumatoid arthritis (RA), the structures of the joint are damaged by the characteristic inflammatory process ${ }^{1}$ The Disease Activity Score (DAS28) considers twenty hand joints in the evaluation of rheumatoid arthritis (RA). While the Health Assessment Questionnaire (HAQ) disability index (DI) (HAQ-DI) is the most frequently used instrument for measuring self-reported physical function in rheumatoid arthritis and considers the ability to dress and groom, get up, eat, hygiene, reach, grasp, situations in which the functional capability of the hand is crucial.

Objectives: To assess the relationship between hand prehensile strength, the DAS28 index and HAQ-DI score in patients with diagnosis of RA.

Methods: The prehensile strength was obtained by the dynamometry method from $105 \mathrm{AR}$ patients, the maximum strength levels in the dominant and non-dominant hand were considered. The Disease Activity Score in 28 joints using the erythrocyte sedimentation rate (DAS28-ESR) and HAQ-DI were recorded.

Results: The maximum prehensile strength, on average, was $14 \mathrm{~kg}$, and the weak force category was more prevalent. The prehensile strength of both hands was negatively correlated with the HAQ-DI score and DAS28 index. In an adjusted logistic regression model, the "weak" strength category of the nondominant hand was associated with "moderate clinical activity" in the DAS28 score $(O R=8.59, p=0.02)$, while the category of "weak" strength of the dominant hand was associated with the presence of "some difficulty" of HAQ-DI score $(O R=4.75, p=0.10)$.

Conclusions: The decrease in prehensile strength represents a marker associated with the DAS28 index and HAQ-DI score in the patient with RA, regardless of age, muscle mass, total fat or body mass. The measurement of the prehensile strength can be a useful and inexpensive tool to be considered in the clinical evaluation of the RA.

\section{REFERENCE:}

[1] Janet L Poole. Hand Function in Rheumatoid Arthritis. Mehmet Tuncay Duruöz A Practical Guide to Assessment. Springer. 2014. [ISBN:978-14614-9448-5]

Acknowledgements: None.

Disclosure of Interest: None declared

DOI: 10.1136/annrheumdis-2018-eular.7534

\section{AB0356 BUILDING A PATIENT-CENTRED CARDIOVASCULAR RISK REDUCTION PROGRAM FOR PATIENTS WITH INFLAMMATORY ARTHRITIS}

I.Y. Navarro-Millan ${ }^{1}$, S. Young ${ }^{2}$, S. Shurbaji ${ }^{3}$, C. McDavid ${ }^{3}$, A. Cornelius-Schecter ${ }^{1}$, B. Johnson ${ }^{3}$, A. Cherrington ${ }^{3}$, L. Fraenkel ${ }^{4}$, J. Curtis ${ }^{3}$, M. Safford ${ }^{1}$. ${ }^{1}$ Weill Cornell Medicine, New York; ${ }^{2}$ Binghamton University, Binghamton; ${ }^{3}$ University of Alabama at Birmingham, Birmingham; ${ }^{4}$ Yale University, New Haven, USA

Background: Cardiovascular disease (CVD) is the most common cause of death among patients with inflammatory arthritis (IA) such as rheumatoid arthritis (RA), psoriatic arthritis (PsA) or ankylosing spondylitis (AS).
Objectives: This study is the formative work to help design a patient-centred intervention for a CVD risk reduction program for patients with IA

Methods: We conducted a qualitative study guided by Bandura's Social Cognitive Theory, placing special focus on knowledge about the relationship between arthritis and CVD as well as barriers and facilitators to receiving healthcare related to CVD risk such as screening and management for hyperlipidemia. Participants had to have either RA, PsA or AS and were recruited from a single academic centre. Data were analysed thematically.

Results: We conducted three focus groups with a total of 17 participants. Mean age of participants was 56 (SD \pm 7.7 ) years; 15 were women; 3 were on a statin and 1 previously had a stroke. The majority of the participants were Five themes emerged: 1) Need for more information about IA and medications; 2) Lack of edu cation about CVD risk and IA; 3) CVD risk reduction as an integrated lifestyle modification; 4) Uses for peer support around relevant CVD risk factor mitigation approaches; and 5) Improving doctor-patient communication about RA. The themes that emerged in our study showed that the majority of these IA patients were not aware of the relationship between CVD and IA, and were very interested in learning about IA, medication side effects, and prognosis of IA. These participants wanted to learn first about IA and, as a secondary goal, learn about their CVD risk in the context of their IA rather than as a new condition.

Conclusions: Providing a clear understanding about systemic effects of IA and how to treat it will be needed before focusing on CVD risk reduction. By doing so, there will be better opportunity for the CVD risk reduction program to succeed. Disclosure of Interest: I. Navarro-Millan: None declared, S. Young: None declared, S. Shurbaji: None declared, C. McDavid: None declared, A. CorneliusSchecter: None declared, B. Johnson: None declared, A. Cherrington Grant/ research support from: Boehringer Ingleheim, Consultant for: Novo Nordisk, Astra Zeneca, L. Fraenkel: None declared, J. Curtis: None declared, M. Safford Grant/ research support from: Amgen, Inc

DOI: 10.1136/annrheumdis-2018-eular.2932

\section{AB0357 1 THE SUPPORT OF MEDICAL CLERKS IN RHEUMATIC DISEASE CLINIC AIDS T2T PRACTICE FOR RHEUMATOLOGISTS AND IMPROVES DISEASE ACTIVITIES OF PATIENTS WITH RHEUMATOID ARTHRITIS}

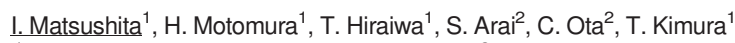
${ }^{1}$ Orthopaedic Surgery, University of Toyama; ${ }^{2}$ Orthopaedic Surgery, Sainou Hospital, Toyama, Japan

Background: With treat-to-target (T2T), the physician always has to evaluate disease activity and joint damage of rheumatoid arthritis (RA) patients exactly to maintain the activities of daily living of the patient for the long term. However, the amount of work required by physicians to complete T2T can be onerous, so the cooperation of medical staff is necessary to practice T2T.

Objectives: The purpose of this study is to clarify the role and effectiveness of medical clerks (MCs) in a rheumatic disease clinic.

Methods: In our rheumatic disease clinic, MCs have supported rheumatologists since April 2011. We individually evaluated 50 RA patients in May 2010 (before MC support: "preceding period"), April 2011 (1 year after the start of MC support: "early period"), April 2013 (3 years after the start of MC support: "middle period"), April 2015 (5 years after the start of MC support: "late period") and April 2017 (7 years after the start of MC support: "last period"). We assessed the prevalence of T2T practice, disease activity, and drug use. When all components of the Simplified Disease Activity Index (SDAI) of patients had been listed in the medical record and radiography of hand and foot joints had been undertaken more than once a year, the medical examination was defined as "T2T practice". Disease activity was assessed using the SDAl and Clinical Disease Activity Index (CDAI).

Results: Prevalence of T2T practice was $50 \%, 86 \%, 94 \%, 100 \%$ and $100 \%$ at preceding, early, middle, late and last periods, respectively. Prevalence of T2T practice increased after the start of MC support. Accordingly, disease activities improved gradually. SDAI remission was $30.8 \%$ in the preceding period, $28.5 \%$ in the early period, $30 \%$ in the middle period, $58 \%$ in the late period and $56 \%$ in the last period, respectively. CDAI remission improved towards the last phase, similar to that seen with the SDAI. The mean dose of methotrexate (MTX) increased gradually towards the last phase, but the prevalence of MTX use did not show a remarkable change. Prevalence of use of biological disease-modifying anti-rheumatic drugs did not increase during the study period.

Conclusions: MC support in rheumatic disease clinics aids T2T practice for rheu matologists. The disease activities of RA patients can be improved by MC support.

Disclosure of Interest: None declared

DOI: 10.1136/annrheumdis-2018-eular.1618

(1)

(2)

